\title{
A Calvary of Human Being in Three Dissident Novels (Comparative Analysis on the Main Characters of the Respective Novels: "Doctor Zhivago", "One Day of Ivan Denisovich", "Odin Mondvalsen".)
}

\author{
Eriola Agalliu
}

\begin{abstract}
Albanian language and literature teacher, Ernest Koliqi French-Albanian college, Tirana, Albania Doctoral studies, Department of Literary Criticism, Centre of Albanological Studies, Tirana, Albania

Email: eagalliu@ernestkoliqi.com, agalliu.eriola@yahoo.com
\end{abstract}

\section{Doi:10.5901/ajis.2013.v2n11p106}

\section{Abstract}

The methodological strategy of this study is to take a comparative approach to analyse the three heroes of the dissident literature. Our analysis will focus on three characters of prominent Russian and Albanian literature: - Yuri Zhivago ("Doctor Zhivago" B. Pasternack), Ivan Denisovich Chuhov ("One day of Ivan Denisovich" A. Solzhenitsyn), Odin Mondvalsen ("Odin Mondvalsen" K. Trebeshina). We will try, through text analysis, to shed light on how these characters in the respective novels are artistically portrayed. Seemingly distant, each of them carries the burden of personal and social drama of their time representing at the same time the anti-hero of socialist realism. Their perspective on the world and life in general, their reactions in front of violence and terror, their struggle for survival, are the key analysis points. From Zhivago (doctor and poet) to Chuhov (simple Russian peasant) and then to Odin Mondvalsen (an imprisoned patient), we see basically the transfiguration of the same social being. Placed side by side, this trinity of characters represents the grotesque human drama under the dictatorship.

"Si un habitant de Mars, ignorant tous des choses de la terre, jugeait les divers pays d'après les livres et revues qui y paraissent, il parviendrait certainement à la conclusion que l'Est est habité par des créatures raisonnables, a la pensée claire, et l'Ouest par des avortons et des dégénérés." 1

Par une telle analogie, Czeslaw Milosz dans son livre: "La pensée captive" analyse la différence entre les démocraties de l'Ouest et les pays communistes de l'Est.

Sans doute, L'Ouest nous donne une image des sociétés mouvementées: des gens qui critiquent, qui font des grèves, qui se lamentent incontestables contre l'Etat. Tout au contraire le communisme de l'Est réside dans des sociétés ou les gens applaudissent avec adoration leurs gouvernants et participent volontairement à des parades pompeuses. On y voit partout des visages uniformément heureux, du système totalitaire.

Derrière les belles façades, se cachait, une autre réalité. La littérature dissidente est née comme réaction contre ce type de sociétés, des régimes communistes. Les dissidentes ont apporté à la littérature soviétique l'Antihéros du Réalisme Socialiste. Ce personnage n'ayant pas des grands idéaux, ne se caractérisait pas, par l'optimisme euphorique. II ne croyait pas en le Parti. Artistiquement parlant, ce protagoniste était incomparable au héros de l'existentialisme ou du roman absurde. Dans la société communiste sa vie était un long calvaire de souffrance. Ce sont ces traits qui ont fait de la littérature dissidente un humanisme, qui élevait le culte de l'homme vrai, de celui qui se positionnait contre la doctrine marxiste et contre la lutte des classes.

Dans cette étude comparative nous allons parler de trois héros de la littérature dissidente. Notre analyse se concentrera sur trois personnages de la littérature russe et albanais:

Iouri Jivago ("Le docteur Jivago" B. Pasternak), Ivan Denissovitch Choukhov ("Une journée d'Ivan Denissovitch" A. Soljenitsyne), Odin Mondvalsen ("Odin Mondvalsen" K. Trebeshina).

"Le Docteur Jivago", est peut-être, le romain le plus lu, de la littérature soviétique du XXe siècle. La narration de Pasternak, correspond chronologiquement à la période entre 1905-1945. Ces quarante années ont marqués le début de l'idéologie marxiste en Russie, transformée plus tard, à la fin du Deuxième Guerre Mondiale, à un système totalitaire. Le héros louri Andréiévitch était un aristocrate, et pour cela qu'il ne pouvait pas être d'accord avec le nouveau régime qui 
prêchait la dictature du prolétariat. En plus en tant que médecin et poète de grand talent, ses principes vont contre l'antihumanisme et la surveillance de l'individu par l'Etat. II est plus patriote que le bolcheviques qui massacrent les russes au nom de la Révolution. louri déteste cette guerre cruelle, incompréhensible, mais en même temps "il aime La Mère-Russie".

Dans le roman, il y a trois aspects de la figure du Docteur Jivago:

- L'intellectuel pacifiste.

- Le docteur humaniste.

- Le poète rêveur.

D'après un communiste, avec qui il discute, la veine qui nourrit la conscience sociale chez louri est atrophiée, ce qui est impardonnable à un médecin cultivé. Dans une conversation avec un des commandants de la Révolution, louri s'exprime:

"Mais tout d'abord le perfectionnement général comme on le comprend depuis Octobre ne m'enthousiasme pas. Deuxièmement, ce ne sont encore que des phrases et on les a déjà payées de tant de sang que, vraiment, le jeu n'en vaut pas la chandelle. Troisièmement, et c'est le principal, lorsque j'entends parler de transformer la vie, je ne me possède plus et je tombe dans le désespoir. "Transformer la vie! Ceux qui parlent ainsi en ont peut-être vu toutes les couleurs, mais la vie, ils n'ont jamais su ce que c'était, ils n'ont jamais senti le soufflé, l'âme. " 2

Donc cela nous permet de dire qu'il partage les mêmes aspirations que les révolutionnaires: II veut le triomphe des valeurs morale et de la justice dans le monde entier. Mais comment cela peut être réalisable? Est-ce qu'il faut inciter les assassinats, les complotes, ou les vengeances primitives? Dans le roman l'auteur décrit des scènes tragiques de la Guerre Civile. La présentation authentique de cette guerre n'a pas à avoir ni avec la fidélité envers le Tsar, ni avec la victoire révolutionnaire, tellement prêchée par Lénine. L'armée blanche et les partisans rouges ne sont que deux groupes qui veulent, avant tout, survivre. Cette guerre n'a absolument rien d'héroïque. Iouri Jivago est le premier qui a compris cela. C'est pourquoi il reste indiffèrent, face à chaque évènement, que cela soit une défaite ou une victoire. Sa mission de médecin à la frontière le fait rester a cote des partisans. Mais il soigne avec la même dévotion les blessures d'ennemis. II hait la lutte et dans des moments de la solitude, il écrit des poésies. Et c'est la poésie qui l'aide à survivre dans cet enfer. Dans une de ces moments, il pense:

“En naissant, tout homme est un Faust qui doit tout embrasser, tout éprouvé, tout exprimé. [...]Qu'est-ce qui m'empêche de faire mon travail de médecin et d'écrire? Je pense que ce ne sont ni les privations, ni notre vie errante, ni les sentiments d'instabilité que me donnent tous ces changements, mais bien l'esprit du temps, cet esprit d'emphase qui est maintenant si répandu: le genre " aube du futur »; "édification d'un monde nouveau »; "flambeaux de l'humanité". Quand on entend ces mots on se dit d'abord: quelle imagination grandiose, quelle richesse! Mais si l'on y regarde de près, l'emphase n'est là que par l'absence de talent.". 3

louri Jivago ne trouve ni repos ni paix dans une société, ou pour survivre, il faut dépourvoir de toute valeur morale. D'une part il lui est impossible de faire des compromis avec le régime, de l'autre part, une rébellion contre la dictature ne serait que le début d'un nouveau calvaire de violence. Et notre héros, désillusionné, pense qu'il ne vaut pas la peine de s'y retourner. C'est la raison pour la quelle la morte est la seule voie de sortie.

$* * *$

Soljenitsyne, c'est le deuxième auteur qui était le témoin de la terreur staliniste, dans les prisons ou souffrent des milliers d'innocents. Son héros s'appelle Ivan Denissovitch Chukhov. Visar Zhiti, un poète albanais qui a souffert dans les prisons communistes albanaises, a écrit dans la préface de l'édition en albanais : "Le roman "Une journée d'Ivan Denissovitch" est un long récit, dans un court roman, pour le crime universel du communisme ».

La terrible douleur de Docteur Jivago, prend ici l'aspect d'un désespoir ironiquement tragique du récit. Ce large tableau de la victoire de la Révolution et des idéaux du Nouveau Monde, chez Soljenitsyne se condense en 24 heures de la vie du prisonnier (du zek) 854, Choukhov, dans une cellule froide. II est un simple paysan d'un esprit russe. Autrefois il a été combattant, mais maintenant il cherche seulement à survivre.

"D'après son dossier, Choukhov est au camp pour trahison de la Patrie. II a fait tous les aveux qu'il fallait: il s'est rendu 
aux Allemands parce qu'il avait envie de trahir l'Union soviétique, et il s'est, soi-disant, évadé parce qu'il avait reçu une mission des services de renseignements de l'ennemi. Quelle mission? Choukhov n'était pas assez futé pour en trouver une. Ni non plus l'officier du contre-espionnage. Alors c'était reste comme ça: "Une mission." Au contre-espionnage, on l'avait salement passé à tabac, Choukhov. Alors il avait réfléchi: s'il ne signait pas, c'était le champ de navets. En signant, il se donnait au moins une chance de vivre encore un peu. II avait signé. " 4

Jusqu'à ce moment-la, il a fait 8 ans de prison et il ne sait pas encore combien il lui en reste, même s'ils l'ont condamné de 10 ans.

II est habitué à cette situation. II n'a pas le temps à penser à ce qui est injuste. Les journées d'Ivan Denissovitch sont toujours les mêmes, comme l'uniforme que le prisonnier (le zek) porte depuis des années. Dans la prison prévaut la loi de la jungle et d'après cette loi, les serviles, les stimulants, et ceux qui veulent espionner a la porte de l'officier opératif y meurent.

II a appris cette horrible vérité depuis longtemps, et c'est pour cette raison qu'après 8 ans, il est encore vivant. II sait comment aider les autres et comment demander leur aide. II a peur des conflits et il sait que même les autres ont peur de lui. Taciturne et patient, il comprend très bien que parler et se taire au bon moment, l'aide à survivre. La logique de Choukhov est simple. II n'y a pas place pour des dilemmes existentialistes: faut-il continuer à vivre dans cet enfer ou non. II sait que cela est difficile, mais non pas impossible. Ivan Denissovitch n'accuse personne. II ne culpabilise pas Dieu du pourquoi sa vie, a pris une telle direction. La prison est si real et si terrifiant, qu'il ne pense plus ni aux causes ou ni aux conséquences.

"Or même pour penser, ça n'est jamais libre, un prisonnier. On retourne toujours au même point, en n'arrêtant pas de retourner les mêmes idées. Est-ce qu'ils ne vont pas retrouver la miche en fourgonnant dans la paillasse? ${ }^{5}[. .$.$] Dans les$ camps et dans les prisons, Ivan Denissovitch s'était déshabitué de prévoir: pour aujourd'hui comme pour dans un an, et comme aussi pour faire vivre les siens. Les chefs s'en occupant à votre place; autant de soucis en moins. " 6

II n'y a rien d'héroïque, dans ce récit. Cette tragi-comédie est juste une petite partie du massacre idéologique qui a fait condamner des milliers d'innocents. Choukhov le sait bien. Et pourtant, après 8 ans, la prison lui semble quelque chose de normale. Dans ces conditions il pense que ni les gardians, ni les investigateurs, ne sont coupables, mais luimême. C'est une sorte de faute qu'il n'avoue jamais, mais elle se réalise par l'ironie du récit. Choukhov a une conviction absurde: le régime est si fort et inébranlable qu'il croit qu'avec le décret spécial du pouvoir soviétique le soleil, maintenant, atteint sa hauteur maximum à une heure et pas à l'heure de midi...

II y a une raison pourquoi l'auteur a choisi un héros comme Choukhov. II n'est pas un intellectuel qui veut réfléchir sur sa vie et sa tragédie. II n'est pas non plus un croyant de cœur chaud prononçant les paroles du Crist. Choukhov représente le russe simple qui croit en des choses pratiques de la vie: la famille, le travail, la campagne. S'il avait tous cela, il serait heureux. La journée d'Ivan Denissovitch a fini de la même manière qu'elle a commencée. (Peut-être, pendant ce jour-là il a été chanceux, parce qu'il n'a eu aucun problème).

$\mathrm{Ca}$ a été pareil hier, ca sera pareil demain. Choukhov sait très bien que 8 ans de sa vie se succèdent d'après ce cycle répétitif. Ce qu'il ne sait pas, c'est quand tout cela va finir?!

Soljenitsyne finit le roman de la même façon qu'il l'a commencé. La première phrase dans son roman est: "A cinq heures du matin, comme tous les matins, on sonna le réveil". Et "Une journée d'Ivan Denissovitch" finit avec cette phrase: "Des journées comme ça, dans sa peine, il y en avait, d'un bout à l'autre, trois mille six cent cinquante-trois. Les trois de rallonge, c'était la faute aux années bissextiles." ${ }^{7}$

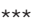

Pourquoi on a choisi deux personnages de la littérature de l'ex - Union Soviétique? Parce qu'ils représentent, essentiellement le drame humain, aux limites de la folie. Le héros albanais Odin Mondvalsen semble avoir affranchi cette

\footnotetext{
${ }^{4}$ Soljenitsyne Alexandre, "Une journée d'Ivan Denissovitch", traduit du russe par Lucia et Jean Cathala, Editions Robert Laffont, S. A, Paris, 2010, ph: 88-89.

${ }^{5}$ Soljenitsyne Alexandre, "Une journée d'Ivan Denissovitch", traduit du russe par Lucia et Jean Cathala, Editions Robert Laffont, S. A, Paris, 2010, ph: 51.

${ }^{6}$ Soljenitsyne Alexandre, "Une journée d'Ivan Denissovitch", traduit du russe par Lucia et Jean Cathala, Editions Robert Laffont, S. A, Paris, 2010, ph: 56.

${ }^{7}$ Soljenitsyne Alexandre, "Une journée d'Ivan Denissovitch", traduit du russe par Lucia et Jean Cathala, Editions Robert Laffont, S. A, Paris, 2010, ph: 226 .
} 
frontière. Entre un intellectuel et un paysan, il est un peu, les deux à la fois.

Les deux auteurs qu'on a mentionnés sont deux nobélisés russe du XXe siècle. Le troisième auteur n'a pas été récompensé à une grande échelle. II est un écrivain oublié, venant d'un petit pays. (Le roman "Odin Mondvalsen" est écrit par Kassem Trebeshina en 1956 mais, malheureusement, il a été publié seulement en 1992 à Priština. Qu'est-ce on pourrait dire si Trebeshina aurait publié son roman en 1956, en Albanie et en Europe? Nous ne pouvons pas dire comment cette œuvre aurait été reçue par la critique, mais ce que nous pouvons dire, c'est que la publication aurait totalement changé l'avenir de la littérature albanaise. Sans doute la plus grande perte de Trebeshina, est le fait: l'édition du œuvre 35 ans plus tard...)

Dans ce trinôme de personnages, Odin Mondvalsen est albanais autant qu'oriental idéologiquement parlant. (Quand je dis 'oriental' je me réfère toujours aux pays de l'Est qui ont vécu le communisme.) Son tragédie commence là où finit le drame de Jivago et la force de Choukhov. Le héros de Trebeshina porte en soi-même, d'une manière incompréhensible, l'indifférence d'louri face à la Révolution et la souffrance d'Ivan Denissovitch dans la cellule de prison. A la différence des deux premiers romans, le récit de Mondvalsen est à la première personne. Mondvalsen a son propre style de confession.

"Auparavant j'avais un nom, et maintenant je m'appelle... Je m'appelle Odin Mondvalsen. Qu'importe aux autres comment je m'appelle?... Vous n'avez jamais entendu ce nom? Très bien: entendez-le maintenant! Comment?... Vous ne voulez-pas entendre? Pourquoi?... Parce que vous ne l'avez jamais entendu? Et alors?... Tu ne l'as jamais entendu avant? Et bien entende-le, maintenant." 8

Dès le début du roman, le narrateur raconte son propre aliénation, entre deux temps, entre ce qui a été et ce qui est d'aujourd'hui. Hier il avait un nom, et aujourd'hui il s'appelle...Odin. (L'auteur ne veut pas nous dire de quel période historique il s'agit entre les mots "auparavant" et "maintenant".) Nous comprenons spontanément, qu'il n'est plus ce qui a été. Même s'il vit dans une prison-psychiatrie, enfermé dans une réalité absurde, Mondvalsen reste l'un des héros les plus authentiques, parmi tous ces fous de la littérature mondiale. II est idiot comme Le Prince Michkino, sincère comme Phommat Gordejeve, bon comme Svejk, étranger et tragique comme Meursault. Au fond tous ces personnages représentent la même chose. Ils dénoncent une société fondée sur la violence, l'indifférence, la guerre et le mort... Ces héros, étrangers à première vue, sont extraordinaires avec tous ce qu'ils sentent et ce qu'ils dissent... Dans un dialogue de roman, Mondvalsen dit:

\begin{abstract}
«Et c'est pour ça que personne n'a le droit de s'élever contre les lois et les règnes, a l'exception des cas où le mal généralisé ne s'étende plus sur le territoire du règne et les armes sont nécessaires pour renverser le vieil ordre sociale. Ici, il faut savoir que la nouvelle loi ne repose pas sur la force des armes, mais sur les intérêts du people qui commence à respirer librement." 9
\end{abstract}

En effet, dans le roman l'esprit malade d'Odin, confonde les pharaons de L'Ancien Egypte, avec les coopératives agricoles (un "succès" du régime totalitaire en Albanie); Scanderbeg (le héros national albanais), avec Le beau Joseph de la Bible: La Danemark où il veut aller, avec la planète Mars où il est arrivé....

En étant fou et non cohérent dans ce qu'il dit, ce personnage est libre à penser et à faire tout ce qu'il veut dans son propre absurde. Etant ainsi, il accuse avec force... La tragédie du héros de Trebeshina est la tragédie d'homme dénaturé à cause de la violence et de la terreur. Le Danemark (détail symbolique dans l'œuvre), est pour lui la Terre promise, où il veut mettre pied à n'importe quel prix. Et pourtant Odin s'exprime:

"La Danemark est vraiment loin, mais je dans mon cœur la morale du peuples européens." 10

Cette idée, répétée sous diverses formes dans le roman, dépeint l'intérieur du héros. Qu'est-ce que cela veut dire avoir au fond de toi la morale des peuples européens? (Un refrain qui se répète depuis 20 années en Albanie). Cela veut

\footnotetext{
${ }^{8}$ Trebeshina Kassem "Legjenda e asaj që iku », Shtëpia Botuese e Lidhjes së Shkrimtarëve, Tiranë, 1992, (mon traduction de l'albanais) ph:81.

9 Trebeshina Kassem, "Legjenda e asaj që iku », Shtëpia Botuese e Lidhjes së Shkrimtarëve, Tiranë, 1992, (mon traduction de l'albanais) ph:112.

10 Trebeshina Kassem "Legjenda e asaj që iku", Shtëpia Botuese e Lidhjes së Shkrimtarëve, Tiranë, 1992, (mon traduction de l'albanais) ph: 150 .
} 
dire que, malgré tout la dictature n'a pas réussi à tuer le rêve d'être libres....

Les personnages de Pasternak, Soljenitsyne et Mondvalsen sont très semblables. Les idéaux et l'esprit poétique d'Iouri Jivago, la patience et le silence titanique de Choukhov ressemblent à l'aliénation de l'être d'Odin. Dans les trois extraits ci-dessous nous lisons:

\begin{abstract}
"Ils ne déclarèrent pas sa disparition (celle de Jivago) au commissariat pour éviter de rappeler aux autorités l'existence d'un homme qui, bien qu'il fut en règle et que son casier judicaire fut vierge, était loin d'être un citoyen exemplaire au regard des idées actuelles. ${ }^{11}$
\end{abstract}

"Tous ceux qui ont fini leur peine pendant la guerre, il y a eu pour eux une disposition spéciale: on les a gardés jusqu'en 1946. De sorte que ceux qui avaient par condamnation trois ans à tirer, ça leur en a fait cinq de pas prévus. La loi, ça se retourne. Tu as fini tes dix ans? On t'explique: "Fais-en encore dix par-dessus le marché. Ou on t'expédie en relégation". 12

"Mais l'hospitalisation de cet homme (de Mondvalsen) n'est pas faite après une arrestation pour avoir commis un crime politique?" 13

Les trois héros sont le produit d'une société où règnent la violence et la haine idéologique. Et précisément parce qu'ils sont des victimes, ils se transforment en même temps des dénonciateurs farouches de la dictature communiste. Homo Soviéticus, annoncé par les slogans du réalisme socialiste n'était que la façade d'une société, en apparence brillante e magnifique. Derrière cette façade, se cachait une réalité sombre et mortelle qui mutilait l'esprit humain par les mécanismes du pouvoir, ledit populaire. C'est ainsi que les trois personnages s'expriment dans de différents extraits:

\begin{abstract}
"Je pense que la collectivisation a été une faute, un échec. On ne pouvait pas l'avouer. Afin de masque l'échec, il a fallu recourir à tous les moyens d'intimidation possible pour ôter aux gens l'habitude de juger et de penser, pour les forcer à voir ce qui n'existait pas et à prouver le contraire de l'évidence. De là la cruauté sans précédent de la terreur [...], la promulgation d'une constitution destine à ne pas être appliquée, l'octroi d'élections qui n'étaient pas fondées sur le principe électoral." 14
\end{abstract}

\begin{abstract}
"A chien battu, montrer le fouet suffit. [...] Des fois, maintenant, qu'on essaye de débrouiller la chose, c'est pour quoi, ces tant-pour-cent? Pour le camp. A cause de ça, le camp tire du chantier des mille et des cent en rabiot, il verse des primes à ses lieutenants [...] et, à nous autres, deux cents grammes de pain le soir en rabiot. Or deux cents grammes, ca régit une existence. ${ }^{15}$
\end{abstract}

"Il n'est pas difficile pour eux de te renvoyer là et de te condamner à une autre peine que tu ne peux même pas imaginer. Leurs punitions sont telles que les vivants envient les morts." 16

Dans les trois exemples mentionnés ci-dessous, nous voyons une mosaïque (ou un puzzle) représentant la vie d'individu dans les conditions d'un tel régime. De Jivago à Choukhov et enfin à Odin Mondvalsen nous apercevons clairement, le même être humain, qui, dans des périodes et pays différents souffre du même drame.

Cette trinité effrayante est le témoignage le plus clair des conséquences de la dictature stalinienne et de ses mauvaises copies, dans tous les pays du Bloc de l'Est. Cet espace de pensée et liberté que nous voyons chez Jivago, se fond avec la force et l'ignorance de Choukhov pour trouver sa plus grande expression dans la folie silencieuse de Mondvalsen.

De la taille tragique du premier romain, au réalisme factuel, presque froid du deuxième romain, nous touchons à

\footnotetext{
${ }_{11}$ Pasternak Boris, "Le docteur Jivago", Edition Gallimard, Paris, 1958, ph : 619.

12 Soljenitsyne Alexandre, "Une journée d'Ivan Denissovitch", traduit du russe par Lucia et Jean Cathala, Editions Robert Laffont, S. A, Paris, 2010, ph: 88 .

13 Trebeshina Kassem "Legjenda e asaj që iku », Shtëpia Botuese e Lidhjes së Shkrimtarëve, Tiranë, 1992, (mon traduction de l'albanais) ph: 125.

14 Pasternak Boris, "Le docteur Jivago", Edition Gallimard, Paris, 1958, ph: 646-647.

${ }^{15}$ Soljenitsyne Alexandre, "Une journée d'Ivan Denissovitch", traduit du russe par Lucia et Jean Cathala, Editions Robert Laffont, S. A, Paris, 2010, ph: 80.

${ }^{16}$ Trebeshina Kasem, "Legjenda e asaj që iku ", Shtëpia Botuese e Lidhjes së Shkrimtarëve, Tiranë 1992 (mon traduction de l'albanais) ph: 87.
} 
un monde absurde et grotesque dans le troisième romain. En fin de compte, quand il n'y a plus d'autre possibilité, la folie demeure le seul refuge sûr, pour pouvoir survivre...

Juri Jivago meurt, Ivan Denissovitch Choukhov vit ses jours uniformes dans la prison, pendant qu'Odin Mondvalsen s'erre entre une prison ou un hôpital psychiatrique. (Le lecteur ne comprend jamais de quelle institution, il s'agit dans l'œuvre). II semble qu'Odin Mondvalsen met fin au cycle ouvert par Jivago plus d'un demi-siècle avant....

En ce qui concerne le phénomène d'intertextualité dans la littérature, Umberto Eco dit:

"C'est important que les livres parlent, entre eux". ${ }^{17}$

Qu'est-ce que cela veut dire, tout ça? Même si Umberto Eco parle ici de l'impact de la culture, nous analyserons cette expression sous un autre point de vue: Les livres parlent entre eux, parce que l'esprit humain parle la même langue, éprouve la même souffrance, fait preuve du même courage, dans la lutte contre du mal et ses formes, d'annihilation du genre humain.

En conclusion, nous pouvons dire que les trois auteurs que nous avons analysés, étant les représentants de la dissidence, dans un système totalitaire, sont semblables entre eux. Docteur Jivago a vécu l'installation du régime rouge en voyant de ses propres yeux les crimes commis au nom de la révolution. Ivan Denissovitch Choukhov endura la férocité des cellules sombres de la dictature. Odin Mondvalsen fut aliéné dans son humanité, sous la violence effrayante de cette prison-psychiatrie appelée Communisme.....

\section{References}

Pasternak Boris, "Le docteur Jivago", Edition Gallimard, Paris, 1958.

Soljenitsyne Alexandre, "Une journée d'Ivan Denissovitch", traduit du russe par Lucia et Jean Cathala, Editions Robert Laffont, S. A, Paris, 2010.

Trebeshina Kasem, " Legjenda e asaj që iku “, Shtëpia Botuese e Lidhjes së Shkrimtarëve, Tiranë 1992.

Pasternak Boris, " Doktor Zhivago ", Uegen, Tiranë 2002.

Sollzhenicin Aleksander, "Një ditë e Ivan Denisovicit ", Albinform, Tiranë 1992.

Chevrel Ives "Letërsia e krahasuar ", Albin, Tiranë 2002.

Eko Umberto " Për letërsinë ", Dituria, Tiranë 2007.

Jeferson An, David Roby, " Teoria letrare moderne ", ALBAS, Tiranë 2006.

Czeslaw Milosz "La pensée captive", Editions Gallimard, Paris, 1953, traduit par A. Prudhommaux et l'auteur.

Milozc Czeslav, "Mendja e robëruar ", Dritëro, Tiranë 1998. 